\title{
LOS DIFERENTES TIPOS DE RESULTADOS EN LAS COOPERATIVAS PORTUGUESAS. UN ESTUDIO DE CASO MÚLTIPLE
}

\author{
POR \\ Ana Maria BANDEIRA ${ }^{1}$, \\ Deolinda MEIRA ${ }^{2}$ y \\ Vera ALVES $^{3}$
}

\section{RESUMEN}

El objetivo de este estudio consiste en identificar los diferentes tipos de resultados en las cooperativas portuguesas, comprender el tratamiento jurídico y contable de los mismos y evaluar si la normativa contable vigente permite constatar sus especificidades.

De este modo, utilizando la metodología cualitativa, mediante el análisis de contenidos y un estudio de caso múltiple, se procede a una reflexión sobre el régimen jurídico y contable de los resultados de las cooperativas.

La información obtenida muestra que el tratamiento jurídico y contable de los resultados no se adecuan debidamente a la realidad de las cooperativas. No son claramente identificables en la legislación vigente los diferentes tipos de resultados, que se someten al mismo tratamiento contable que los resultados de las sociedades mercantiles a pesar de las diferencias sustanciales entre ambas formas jurídicas.

Así pues, defendemos la alteración de la normativa contable aplicable a las cooperativas para que se las someta a la normativa aplicable a las entidades sin ánimo de lucro, de forma a que se evidencie el objeto real de estas, principalmente su finalidad

\footnotetext{
${ }^{1}$ Profesora Adjunta de Contabilidad Financeira en el Instituto Politécnico do Porto/ISCAP. Investigadora del CECEJ (Centro de Estudos em Ciências Empresariais e Jurídicas) y del CEPESE (Centro de Estudos da População, Economia e Sociedade). Correo electrónico: bandeira@iscap.ipp.pt.

${ }^{2}$ Profesora Adjunta de Derecho Mercantil en el Instituto Politécnico do Porto/ISCAP. Investigadora del CECEJ(Centro de Estudos em Ciências Empresariais e Jurídicas) y del SGECOL (Study Group on European Cooperative Law). Correo electrónico: meira@iscap.ipp.pt.

${ }^{3}$ Investigadora del Instituto Politécnico do Porto.

REVESCO No 123 - Primer Cuatrimestre 2017 - ISSN: 1885-8031 - www.ucm.es/info/revesco
}

http://dx.doi.org/10.5209/REVE.54919

Fecha de recepción: 12/04/2016

Fecha de aceptación: 28/10/2016 
mutualista, lo cual permitirá establecer diferencias contables entre los diferentes tipos de resultados, dado que el modelo de las demostraciones incluidas permitirá discriminar los resultados según las diversas actividades.

Palabras clave: Cooperativas, Excedentes, Retorno, Beneficios, Resultado neto.

Claves Econlit: M41, M19, L31.

\title{
DIFFERENT TYPES OF RESULTS IN THE PORTUGUESE COOPERATIVE. MULTIPLE CASE STUDY
}

\begin{abstract}
The aim of this study is to identify the different types of results in the Portuguese cooperative, understand the legal and accounting treatment of them and assess whether the accounting standards allows to highlight the specificities of the results.

Using a qualitative methodology, using content analysis and a multiple case study, we study the legal and accounting regime of the results of cooperatives.

We find that the legal and accounting treatment of the results is not properly suited to the reality of cooperatives. There are no clearly identifiable in the current legislation the different types of results, which are subject to the same accounting treatment results of commercial companies, despite substantial differences between the two legal forms.

Thus, we advocate the change of accounting framework applicable to cooperatives, subjecting them to the normative applicable to non-profit organizations, in order to highlight the real object of these, namely its mutualistic scope, which will differentiate, in the accounting documents, the different types of results, as the model of the statements contained therein allows the deployment of the results by activities.
\end{abstract}

Keywords: Cooperatives, Surpluses, Return, Profit, Net Income. 


\section{INTRODUCCIÓN}

De acuerdo con el art.2 $2^{\circ}$ nº del Código Cooperativo Portugués (CCoop ${ }^{4}$ ) "las cooperativas son personas jurídicas autónomas, de libre constitución, de capital y composición variables que, a través de la cooperación y la ayuda mutua entre sus miembros, con obediencia a los principios cooperativos, persiguen, sin ánimo de lucro, la satisfacción de las necesidades y aspiraciones económicas, sociales y culturales de aquellos."

Esta noción se apoya en cuatro características particulares de este tipo de personalidad jurídica: las dos primeras de carácter formal (la variabilidad del capital social y la variabilidad de la composición societaria) y las restantes de naturaleza sustantiva - el objeto de la cooperativa (la satisfacción, sin ánimo de lucro, de las necesidades económicas, sociales o culturales de los miembros) y el modo de gestión de la empresa cooperativa (la obediencia a los Principios Cooperativos 5 y a la cooperación y ayuda mutua entre sus miembros).

Las cooperativas tienen una finalidad mutualista, dado que su objeto se centra en la satisfacción de las necesidades de sus miembros. Las cooperativas están formadas por personas que quieren cooperar entre sí o, más específicamente, que quieren vender, trabajar, consumir o prestar servicios conjuntamente. Para cumplir este propósito, constituyen una entidad jurídica en la cual realizan dichas actividades.

Asimismo, toda la actividad social de la cooperativa se orienta hacia sus miembros, que son los destinatarios principales de las actividades económicas y sociales que ésta lleva a cabo y en la cual los miembros participan (Fajardo García, 1997; Vargas Vasserot, 2006). Denominaremos "actividad cooperativizada" a esta actividad. Esta, según Vargas Vasserot (2006), se materializa en un conjunto de operaciones en las que se cumplen tres circunstancias, a saber: que sean operaciones internas, es decir, que ocurran en el ámbito de la cooperativa; que sean realizadas por el cooperativista con la cooperativa o viceversa; que estén íntimamente relacionadas con la consecución del objeto social de la cooperativa.

\footnotetext{
${ }^{4}$ Ley 119/2015, de 31 de agosto, que aprobó el Código Cooperativo y revocó la Ley n. $51 / 96$ de 7 de septiembre.

${ }^{5}$ Los principios cooperativos están consagrados en el art. $3^{\circ}$ del Ccoop y corresponden con los siete principios cooperativos definidos por la Alianza Cooperativa Internacional: $1^{\circ}$ Adhesión voluntaria y libre; $2^{\circ}$ Gestión y control democrático por los miembros; $3^{\circ}$ Participación económica de los miembros; $4^{\circ}$ Autonomía e independencia; $5^{\circ}$ Educación, formación e información; $6^{\circ}$ Cooperación entre cooperativas; $7^{\circ}$ Interés por la comunidad.
} 
En ese sentido, el art. $22^{\circ}, n^{\circ} 2$, pár. c) del Ccoop establece que los cooperativistas deben "participar en general en las actividades de la cooperativa y prestar el trabajo o el servicio que les competa”. Se establece por tanto una relación jurídica compleja, en la cual es destacable, por un lado, la obligación asumida por el cooperativista de participar en la actividad de la cooperativa y, por otro lado, la contraprestación realizada por esta.

De hecho, las cooperativas no tienen como objetivo principal la obtención de beneficios distribuibles, como ocurre en las sociedades mercantiles, sino que a través del esfuerzo propio y la ayuda mutua entre sus miembros se pretende la satisfacción de las necesidades económicas y sociales de estos.

Así, el objeto social de la cooperativa, íntimamente relacionado con la finalidad mutualista, repercute en los tipos de resultados obtenidos.

A los resultados provenientes de la actividad económica desarrollada entre la cooperativa y sus cooperativistas los denominamos excedentes si son positivos, y pérdidas si son negativos. A ellos dedicaremos, más adelante, un análisis pormenorizado.

Sin embargo, aunque se centre en sus miembros, la cooperativa puede, de forma limitada, desarrollar operaciones con terceros (Vargas Vasserot, 2006).

A este respecto, el art. $2^{\circ}, n^{\circ} 2$ del Ccoop establece que "las cooperativas, en la consecución de sus objetivos, podrán realizar operaciones con terceros, sin perjuicios de eventuales límites fijados por las leyes propias de cada rama”. Aunque la ley no defina lo que se debe entender por "terceros", existe consenso doctrinal en que, como afirma Rui Namorado, "terceros, de un punto de vista cooperativo, son todos aquellos que mantengan con una cooperativa relaciones que se encuadren en la consecución de su objeto principal, como si fuesen miembros, a pesar de que de hecho no lo sean" (Namorado, 2005: 184). Tal significa que las actividades con terceros de las que habla el legislador se reportarán a actividades del mismo tipo que las desarrolladas con los cooperadores (Meira, 2010). Por tanto, de lo expuesto resulta que las operaciones con terceros están comprendidas en el objeto social de la cooperativa. Estos resultados provenientes de las operaciones con terceros se denominan resultados extracooperativos.

Se podrá identificar un tercer tipo de resultados, los llamados resultados extraordinarios provenientes de actividad ajena al objeto social de la cooperativa (Fajardo 
García, 1997; Iturrioz (1999). Imaginemos que una cooperativa agrícola arrienda uno de sus almacenes al Ayuntamiento o invierte en acciones de una sociedad anónima. Todas estas operaciones pueden generar beneficios o pérdidas provenientes de operaciones ajenas a la actividad de la cooperativa, que consiste en comercializar los productos agrícolas de sus cooperadores.

El Ccoop no consagra expresamente estos tres tipos de resultados, a diferencia de otros ordenamientos jurídicos, en particular el español. De hecho, esta distinción está expresamente prevista en la legislación española, más específicamente en el artículo $57^{\circ}$, de la Ley Estatal de Cooperativas-Ley 27/1999, de 16 de julio (Carreras Roig, 2011).

Pasaremos en seguida al análisis del régimen jurídico y contable de cada uno de estos tipos de resultados.

\section{RESULTADOS POSITIVOS COOPERATIVOS: LOS EXCEDENTES Y SU RETORNO}

Cuando son positivos, los resultados provenientes de las transacciones con los miembros (los llamados resultados cooperativos) se denominan excedentes.

Así pues, los excedentes son el resultado de las transacciones de la cooperativa con sus miembros y, en consecuencia, pueden ser un valor provisionalmente pagado en exceso por los cooperativistas, o bien un valor pagado en defecto por la cooperativa a los cooperativistas, como contrapartida de la participación de estos en la actividad de la cooperativa. (Fajardo García, 1997; Meira, 2012a).

Un porcentaje del excedente del ejercicio, resultante de las transacciones con los cooperativistas, revertirá en la reserva legal (art. $96^{\circ}, \mathrm{n}^{\circ} 2$, del Ccoop) y en la reserva de educación y formación cooperativa (art. 97, $\mathrm{n}^{\circ} 2$, par. b), del Ccoop), así como en eventuales pagos de intereses por los títulos de capital (art. $100^{\circ}, \mathrm{n}^{\circ} 1$, del Ccoop) (Meira, 2011).

El Ccoop prevé el retorno de parte de los excedentes a los cooperativistas (art. $100^{\circ}$ del Ccoop). El retorno es el instrumento técnico de atribución al cooperativista del excedente, que funciona como una corrección a posteriori, a través de la cual se devolverá a quien haya generado un excedente la diferencia entre el precio practicado y el coste, o la diferencia entre los ingresos netos y los adelantos laborales pagados. Tales diferencias son calculadas con exactitud al final de cada ejercicio (Meira, 2012b; SGECOL, 2015). 
Según el art. $100^{\circ}, \mathrm{n}^{\circ} 1$, del Ccoop, "los excedentes anuales netos, con excepción de los provenientes de las transacciones realizadas con terceros, que quedan después de los eventuales pagos de intereses por los títulos de capital y de la dotaciones para las diversas reservas, podrán retornar a los cooperativistas”. Así pues, el Ccoop prevé la distribución de los excedentes resultantes de las transacciones de la cooperativa con los cooperativistas, con excepción de los provenientes de las transacciones con terceros.

Sin embargo, según el art. $100^{\circ}, \mathrm{n}^{\circ} 2$ del Ccoop "no puede procederse al reparto de excedentes entre los cooperativistas, ni crear reservas libres, antes de que se hayan compensado las pérdidas de los ejercicios anteriores o, habiéndose utilizado la reserva legal para compensar esas pérdidas, antes de que se haya reconstituido al nivel anterior al de su utilización”. En caso de que así ocurra, estaremos ante excedentes ficticios.

Los cooperativistas que reciban excedentes ficticios estarán obligados a restituirlos, a menos que hayan actuado de buena fe cuando los recibieron, teniendo en cuenta lo dispuesto en el art. $34^{\circ}, \mathrm{n}^{\circ} 1$, del Código portugués de Sociedades Mercantiles (CSC), aplicable por remisión del art $9^{\circ}$ del CCoop ${ }^{6}$.

De este modo, el retorno cooperativo consistirá en la parte de los excedentes repartibles que la Junta general decida distribuir entre los cooperativistas. Para ello es esencial que haya una decisión cuyo fin sea el de promover la respectiva distribución. Esta decisión deberá adoptarse en la asamblea de aprobación de cuentas.

La distribución del retorno entre los cooperativistas se hará en función y de forma proporcional a las actividades o transacciones efectuadas con la propia cooperativa (según el valor de las compras o servicios consumidos o prestados, en el caso de cooperativas de consumo o servicios; el valor de transacciones efectuadas o productos entregados, en el caso de las cooperativas agrícolas o de comercialización), o en función y de forma proporcional al trabajo de cada miembro, (como es el caso de las cooperativas de trabajo, donde en la distribución del excedente generado por los miembros deberán ser deducidos los importes ya recibidos "por cuenta de los mismos").

\footnotetext{
${ }^{6} \mathrm{El}$ art. $9^{\circ}$ del Ccoop dispone que "Para colmatar las lagunas del presente reglamento, que no lo puedan ser por recurso a la legislación complementaria aplicable a las diversas ramas del sector cooperativo, puede recurrirse, en la medida en que no se infrinjan los principios cooperativos, al Código das Sociedades Comerciais, especialmente a los preceptos aplicables a las sociedades anónimas.
} 


\section{$\begin{array}{llll}\text { 3. RESULTADOS EXTRACOOPERATIVOS Y RESULTADOS } & Y \text { Y }\end{array}$ EXTRAORDINARIOS}

Más allá de los resultados cooperativos, que son los resultados típicos de las cooperativas, son identificables los resultados provenientes de las transacciones con terceros, que denominamos resultados extracooperativos, los cuales si son positivos reciben también el nombre de beneficios.

De hecho, los resultados positivos provenientes de las transacciones con terceros son beneficios y, por eso, no pueden retornar a los cooperativistas según el art. $100^{\circ}, \mathrm{n}^{\circ} 1$, del Ccoop. Esos beneficios revertirán obligatoriamente en reservas irrepartibles, en los términos dispuestos en los arts $100^{\mathrm{a}}, \mathrm{n}^{\mathrm{o}} 1$ y $99^{\circ}$ del Ccoop. El mismo régimen se aplica a los resultados provenientes de actividades extraordinarias.

Como la cooperativa no tiene ánimo de lucro, sino que pretende la satisfacción de las necesidades de los cooperativistas, esos beneficios no pueden ser repartidos entre los miembros, y deben ser transferidos íntegramente a las reservas irrepartibles. En consecuencia, no son distribuibles (Donário, 2010).

En este contexto, creemos oportuno realzar la necesidad de una contabilización separada de los resultados que permita distinguir los excedentes (resultantes de las actividades cooperativas), de los beneficios (resultantes de las actividades extracooperativas y extraordinarias) (Romero Civera \& Mari Vidal, 2015), como veremos más adelante.

\section{RESULTADOS NEGATIVOS}

En el cierre de cuentas, cuando los gastos ${ }^{7}$ superan a los ingresos ${ }^{8}$, las cooperativas podrán depararse con resultados negativos.

¿De qué forma se podrán subsanar los resultados negativos?

En las cooperativas, esta operación está sometida a reglas específicas.

\footnotetext{
${ }^{7}$ Según el SNC (Estrutura Conceptual §76) Gastos, engloba las perdidas así como aquellos gastos que sean ocasionados por actividades corrientes (u ordinarias) de la entidad. Los gastos que deriven de las actividades ordinarias de la entidad incluyen, por ejemplo, el coste de las ventas, los salarios y las depreciaciones. Generalmente toman la forma de un exflujo o merma de activos tales como el dinero y sus equivalentes, existencias o activos fijos tangibles.

${ }^{8}$ Ingresos, según el $\$ 72$ de la Estrutura Conceptual engloba tanto los réditos como las ganancias. Los réditos provienen de las actividades corrientes (u ordinarias) de una entidad y pueden incluir un sinfín de nombres diferentes, entre los cuales: ventas, honorarios, interesas, dividendos, royalties o rentas.
} 
Teniendo en cuenta lo dispuesto en el Ccoop, habrá que distinguir entre la responsabilidad de los cooperativistas con respecto a las deudas de la cooperativa (responsabilidad externa) y la responsabilidad del cooperativista por sus pérdidas ante la cooperativa (responsabilidad interna).

A propósito de la responsabilidad de los cooperativistas con respecto a las deudas de la cooperativa (responsabilidad externa), el art. 23 del Ccoop, integrado, en la versión actual del Código, en el Capítulo III "Miembros", establece que esta "se limita a la cantidad del capital suscrito, sin perjuicio de que los estatutos de la cooperativa puedan determinar que la responsabilidad de los cooperativistas sea ilimitada, o limitada con relación a unos e ilimitada con respecto a otros".

Por otro lado, el $\mathrm{n}^{\mathrm{o}} 1$ del art. $80^{\circ}$ de Ccoop establece que "sólo el patrimonio de la cooperativa responde por las deudas de esta ante los acreedores".

De estas normas resulta que la responsabilidad de los cooperativistas estará limitada al valor del capital suscrito por ellos, por lo que sólo el patrimonio de la cooperativa responderá por las deudas de la misma.

Sin embargo, la ley admite que los estatutos de cada cooperativa puedan determinar que la responsabilidad de los cooperativistas, o de algunos de ellos, sea ilimitada. De este modo, la responsabilidad ilimitada de los cooperativistas significará una garantía adicional para terceros que contraigan compromisos con la cooperativa, aumentando, por eso, los medios de salvaguarda de los acreedores de la cooperativa.

La responsabilidad interna está prevista en el art. $96^{\circ}, \mathrm{n}^{\circ} 5$ del Ccoop, que prevé la imputación al cooperativista de pérdidas en la proporción de las operaciones, servicios o actividades realizadas por cada uno de ellos con la cooperativa. Atendiendo al criterio de participación en las pérdidas indicado por el legislador (proporción de las operaciones, servicios o actividades realizadas por cada uno de ellos con la cooperativa), se estará hablando de pérdidas resultantes de la participación del cooperativista en la actividad cooperativa. Así, estas pérdidas, originadas en el ejercicio de una actividad realizada por cuenta del cooperativista, deben ser imputadas a los propios cooperativistas, siempre de forma proporcional a su participación en dicha actividad ( $\operatorname{art.} 96^{\circ}, \mathrm{n}^{\circ} 5$ del Ccoop). Por el contrario, las pérdidas producidas en la actividad con terceros y todas las demás pérdidas serán pérdidas sociales, que deben ser solventadas exclusivamente por el patrimonio social (art. $23^{\circ}$ del 
Ccoop). Estas pérdidas son consideradas deudas por la legislación española, a fin de que se distingan de las pérdidas generadas por la actividad de la cooperativa con sus cooperativistas (Marín-Sánchez, et al. 2012; Meira, 2015).

El principio de la responsabilidad limitada del cooperativista por las deudas sociales (art. 23 del Ccoop) no impedirá, por eso, que éste deba hacerse cargo de las pérdidas originadas en el ejercicio de una actividad económica realizada por cuenta del cooperativista, de modo personal y proporcional a su participación en la actividad cooperativizada.

Este régimen de imputación de pérdidas al cooperativista (que está integrado en sus obligaciones económicas) representa una característica singular de las cooperativas.

Veremos más adelante cómo trata el Sistema de Normalización Contable (SNC) ${ }^{9}$ esta cuestión de las pérdidas y si recoge esta distinción entre deudas y pérdidas, típica de las cooperativas.

\section{REGISTRO CONTABLE DE LOS RESULTADOS EN LAS COOPERATIVAS}

La circunstancia de que las cooperativas presenten transacciones con miembros y transacciones con terceros impone una contabilización separada de los resultados, indispensable para que la contabilidad de las cooperativas establezca las diferencias entre los tipos de resultados generados. Sin embargo, la ley no exige actualmente esa contabilización separada, aunque en el pasado el ya revocado Estatuto Fiscal Cooperativo ${ }^{10}$ apuntase en esa dirección. De hecho, el $n^{\circ} 4$, de la Ley no 85/98, de 16 de diciembre, establecía que "la contabilidad de las cooperativas deberá estar organizada de acuerdo con la normalización de la contabilidad y con otras disposiciones legales vigentes para el respectivo sector de actividad y deberá reflejar todas las operaciones realizadas, permitiendo determinar claramente los resultados de las mismas y las variaciones patrimoniales sujetas a los diferentes regímenes de tributación”. Esta contabilización separada sería condición necesaria para un tratamiento fiscal adecuado de los resultados de las cooperativas (Aguiar, 2014).

\footnotetext{
${ }^{9}$ Aviso n. ${ }^{\text {15 }}$ 15652/2009 de 7 de septembre. Diário da República n. ${ }^{\circ}$ 173/2009 - II Série. Secretario-General. Lisboa.

${ }^{10}$ Revocado por la Ley n. ${ }^{\circ} 64-\mathrm{B} / 2011$ de 30 de diciembre.
} 


\subsection{Marco normativo contable de las cooperativas}

A pesar de que son entidades sin ánimo de lucro, las cooperativas, según el DecretoLey (DL) no 158/2009, de 13 de julio, están obligadas a adoptar el SNC. Asimismo, las cooperativas no gozan de un tratamiento contable diferenciado frente a las sociedades mercantiles.

De hecho, el SNC, según el no 1 pár. e) del art. $3^{\circ}$, es de aplicación obligatoria a las cooperativas, lo que ha generado alguna controversia doctrinal y en el movimiento cooperativo, ya que el SNC fue elaborado sobre todo para las sociedades mercantiles ${ }^{11}$, sin tener en cuenta las especificidades de las cooperativas, en particular su finalidad mutualista y el carácter variable de su capital social, derivado del derecho de reembolso de las entradas, en caso de dimisión del cooperativista.

Las sociedades mercantiles tienen como principal objetivo la maximización del beneficio, mientras que el objetivo principal de las cooperativas es la satisfacción de las necesidades económicas, sociales y culturales de sus miembros y no la obtención de lucro (Rodrigues, 2010).

Según la Estructura Conceptual (EC) del SNC los estados financieros deben reflejar una imagen verdadera y apropiada, proporcionando información acerca de la posición financiera, del desempeño y de las alteraciones en la posición financiera de una entidad. Esta información le podrá ser útil a cualquier entidad o persona interesada en la toma de decisiones económicas de la cooperativa.

Sin embargo, estas personas y entidades interesadas mencionadas por la EC, son los potenciales y actuales inversores, la plantilla, los prestamistas, los proveedores y otros acreedores, los clientes, el gobierno y sus departamentos y el público en general. Cabe destacar que la EC no se refiere en ninguna ocasión a los cooperativistas.

Además, los estados financieros en las sociedades mercantiles se elaboran para que quede visible su desempeño económico-financiero, o sea el beneficio, y la forma en la que se reparten los resultados entre los inversores.

\footnotetext{
${ }^{11}$ En términos del $n .^{\circ} 1$ del art. $3^{\circ}$ del DL n ${ }^{\circ} 158 / 2009$ de 13 de julio, el SNC se aplica obligatoriamente a las siguientes entidades: a) Sociedades incluidas en el Código das Sociedades Comerciais; b) Empresas Individuales reguladas por el Código Comercial; c) Establecimientos individuales de responsabilidad limitada; d) Empresas públicas; e) Cooperativas; f) Agrupamientos complementarios de empresas y agrupamientos europeos de interés económico.
} 
Así pues los estados financieros no se ajustan a la realidad de las cooperativas, puesto que no revelan su lógica, basada en una finalidad mutualista, en la cual los únicos resultados susceptibles de reparto son los excedentes y no los beneficios. Este reparto es proporcional a las transacciones realizadas por cada miembro y no a su participación en el capital social (Meira \& Ramos, 2014).

Debemos subrayar que en Portugal las "entidades sin ánimo de lucro" tienen una normativa contable específica incluida en DL n ${ }^{\circ}$ 36-A/2011, de 8 de Marzo, el SNC de las Entidades del Sector No Lucrativo (SNC-ESNL). Sin embargo, el $n^{\circ} 2$ art. $5^{\circ}$ de dicho reglamento excluye expresamente a las cooperativas de su ámbito de aplicación, con excepción de las cooperativas “cuya rama específica no permite bajo ninguna forma, directa o indirecta, el reparto de excedentes, especialmente en las cooperativas de iniciativa social" (Lopes, 2015, pp 155).

Esta aplicación del SNC a las cooperativas se mantuvo en el recientemente publicado DL n 98/2015 de 2 de junio, que alteró el SNC. La posición del legislador no se comprende, puesto que las cooperativas no tienen ánimo de lucro, por lo menos a título principal, ni distribuyen ganancias económicas o financieras directas a sus respectivos cooperativistas.

\subsection{Resultado neto del período}

En ese contexto, consideramos oportuno mencionar la noción de resultado neto del período.

Según la EC, para calcular el resultado neto del período, deberemos tener en cuenta el resultado de su contabilidad.

Este es el resultado global positivo o negativo agregado, de un período económico, como ha sido relatado en la "cuenta de resultados" por naturalezas; antes de la deducción de los respectivos impuestos sobre los beneficios o la adición del respectivo ahorro del impuesto sobre beneficios.

Para ese cálculo debemos tener en cuenta que los resultados corresponden a los réditos y a las ganancias, previstos en los $\S \S 72$ a 75 de la EC, substrayendo los gastos, referidos en los $\S 76$ a 78 de la EC. De esta forma obtenemos el resultado del período. 
Cabe reparar que la EC en ninguna ocasión hace referencia explícita a los beneficios cooperativos, es decir, a los beneficios resultantes de los resultados extracooperativos y extraordinarios, tal y como fue referido anteriormente. De igual modo, la EC tampoco menciona los resultados cooperativos que no son beneficios, o sea los excedentes. Todo esto demuestra que el SNC no se ajusta a la realidad de las cooperativas (Rodrigues, 2010).

Según el SNC en el § 102 pár. a), el beneficio "sólo se obtiene si la cuantía financiera (o dinero) de los activos netos al final del período excede la cuantía financiera (o dinero) de los activos netos del comienzo de período, después de excluirse cualquier distribución a, y contribuciones de, los propietarios durante el período". La misma normativa contable refiere en el $\S 102$ pár. b) que se obtendrá beneficio "si la capacidad física productiva (o capacidad operacional) de la entidad (o los recursos o los fondos necesarios para conseguir esa capacidad) excede al final del período la capacidad física productiva del comienzo del período, después de excluirse cualquier distribución a, y contribuciones de, los propietarios durante el período".

En suma, según el SNC, el beneficio contable es el resultado de un período antes de la deducción del gasto del impuesto sobre el beneficio tributable ${ }^{12}$.

El beneficio tributable o la pérdida fiscal corresponden respectivamente al beneficio o a la pérdida de un período, calculado según las reglas establecidas por las autoridades fiscales, sobre el cual, respectivamente, se pagan o se recuperan los impuestos sobre la renta.

Respecto a las pérdidas, según la EC $§ 77$ estas “representan otros ítems que cumplen la definición de gastos y pueden, o no, surgir en el transcurso de las actividades ordinarias de la entidad. Las pérdidas representan disminuciones en beneficios económicos y como tal no son en su naturaleza diferentes de otros gastos".

Se puede comprobar que este concepto incluido en la EC no se adecua a la realidad de las cooperativas, puesto que no refleja la distinción mencionada con anterioridad entre deudas y pérdidas.

\footnotetext{
${ }^{12}$ Beneficio tributable $=$ Beneficio líquido contable + costes no aceptados fiscalmente + crédito de impuesto desgravable + variaciones patrimoniales positivas - plusvalías a reinvertir en los próximos dos años - beneficios distribuidos en los términos del art. 48 n. ${ }^{\circ} 1$ del Código do Imposto Sobre los rendimientos de entidades variaciones patrimoniales negativas -gratificaciones por cuenta del resultado de este ejercicio. Información disponible en: http://www.iapmei.pt/iapmei-art-03.php?id=510. (consulta realizada el 14 de septiembre de 2014).
} 
En las cooperativas no existen socios sino cooperativistas y para estos las contribuciones de capital tienen un carácter meramente instrumental. Aunque para ser

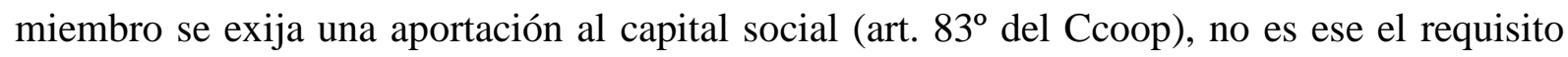
esencial. En efecto, para convertirse en cooperativista y adquirir los correspondientes derechos y obligaciones, es necesario participar en la actividad de la cooperativa, obligación que se relaciona con la mencionada finalidad mutualista de la cooperativa. La posición del cooperativista en la cooperativa estará determinada sobre todo por su participación en la actividad de la cooperativa. Esta participación tendrá un papel relevante en el régimen económico de la cooperativa al servir de parámetro de participación en los excedentes, tal y como ya se ha visto.

\subsection{Resultado distribuible en las cooperativas}

Cuando se habla de resultados distribuibles en las cooperativas, se hace referencia solamente a los excedentes, dado que los beneficios no son distribuibles.

Asimismo, en las cooperativas debemos tener en cuenta el art. $100^{\circ} \mathrm{n}^{\circ} 2$ del Ccoop, que recoge lo siguiente: "no se puede proceder al reparto de excedentes entre los cooperativistas, ni crear reservas libres, antes de que se hayan compensado las pérdidas de los ejercicios anteriores o, habiendo utilizado la reserva legal para compensar esas pérdidas, antes de que se haya reconstituido la reserva hasta el nivel anterior al de su utilización".

Debemos señalar que el régimen jurídico de los excedentes, como figura en el Ccoop, se asemeja al régimen jurídico de los beneficios y los dividendos, ya que el art. $9^{\circ}$ del Ccoop, relativo al derecho subsidiario, remite al CSC. Esta remisión deberá, sin embargo, reunir dos condiciones: por un lado, no incumplir los principios cooperativos y, por otro lado, dentro del espacio constituido por el CSC deberá darse prioridad a los preceptos aplicables a las sociedades anónimas (Fraga \& Gonçalves, 2009).

Como vimos, en las cooperativas los excedentes resultantes de las transacciones con los cooperativistas podrán retornar a estos, así como en las sociedades mercantiles el beneficio también podrá ser distribuido entre los socios bajo la forma de dividendos. Sin embargo, estos dos conceptos presentan diferencias sustanciales.

Así pues, los retornos no son beneficios sociales distribuibles sino excedentes, es decir, ventajas mutualistas generadas por la gestión cooperativa, directamente a favor de los 
cooperativistas, como ya vimos. Los dividendos, a su vez, son una parte de los beneficios sociales que se distribuyen entre los socios (Meira, 2012c).

Otra diferencia que debe ser subrayada es el modo de reparto del retorno entre los cooperativistas. De hecho, en las sociedades mercantiles, los dividendos se reparten entre los socios en proporción a la participación de cada uno en la sociedad, mientras que en las cooperativas el reparto del retorno es proporcional a la participación del cooperativista en la actividad de la cooperativa.

Finalmente, debemos señalar que en las cooperativas el retorno se genera gracias a los propios cooperativistas, mientras que en las sociedades mercantiles la ventaja económica se genera a costa de terceros (Meira, 2012c).

\subsection{Adopción del SNC-ESNL como una vía adecuada para el marco normativo contable de los resultados en las cooperativas}

Como vimos, los estados financieros de las cooperativas son elaborados según el SNC. Sin embargo, las cooperativas son entidades sin ánimo de lucro, reguladas por una normativa específica, el Ccoop.

¿Cuál es el motivo para que las cooperativas no apliquen el SNC-ESNL?

Las $\mathrm{ESNL}^{13}$ presentan un marco contable propio, con reglas específicas definidas en el DL n³6-A/2011, de 9 de marzo. El art. 5º de la referida norma limita su ámbito de aplicación a las entidades que desarrollen a título principal una actividad sin ánimo de lucro y que no puedan distribuir entre sus miembros o contribuyentes cualquier ganancia económica o financiera directa.

Este reglamento excluye expresamente a las cooperativas, con excepción de aquellas cuya rama específica no permita bajo ninguna forma, directa o indirecta, el reparto de excedentes, en particular a las cooperativas de Iniciativa Social, según el art. $5^{\circ}, n^{\circ} 3$, del referido reglamento.

Así pues, el requisito impuesto por el legislador es que las cooperativas no puedan "distribuir entre sus miembros o contribuyentes ninguna ganancia económica o financiera

${ }^{13}$ ESNL- Asociaciones, fundaciones y entidades públicas de tipo asociativo sin ánimo de lucro. 
directa". Ahora bien, como vimos anteriormente, lo que las cooperativas pueden distribuir a los cooperativistas son los excedentes, los cuales no pueden ser considerados "ganancias económicas o financieras directas", sino valores provisionalmente pagados, ya sea en exceso por los cooperativistas a la cooperativa, o en defecto (pérdidas) por la cooperativa a los cooperativistas. En este contexto, no vemos ningún impedimento para que las cooperativas no sean incluidas en esta normativa contable (Bandeira \& Meira, 2015).

Así pues, la aplicación del SNC-ESNL a las cooperativas permitiría evidenciar la diferente tipología de resultados presentes, dado que según las notas del Plan Contable de la clase 8-Resultados, estipuladas en el Decreto 218/2015, de 23 de julio, “en las ESNL podrá ser conveniente discriminar el resultado neto del período según proyectos, valores, o actividades".

En suma, consideramos que el SNC-ESNL es el sistema más adecuado a la realidad de las cooperativas, puesto que los estados financieros de estas proporcionarían una imagen verdadera y apropiada de su desempeño.

\section{METODOLOGÍA: ESTUDIO DE CASO MÚLTIPLE}

Esta sección se refiere a la metodología de investigación utilizada y presenta los principales argumentos tomados en consideración para su elección, de forma que se alcancen los objetivos propuestos.

La metodología de investigación para comprobar cuál es el tratamiento contable de los resultados de las cooperativas será la metodología cualitativa, basándonos en el estudio de caso múltiple.

La elección de un estudio de caso múltiple se debe a que lo consideramos el más adecuado a nuestro estudio, dado que buscamos investigar, comprender y describir los hechos en las cooperativas.

Así pues, con el objetivo de alcanzar nuestro objetivo, fueron elaboradas las siguientes preguntas:

P1: ¿La cuenta de resultados pone de manifiesto los diferentes tipos de resultados?

P2: ¿Cuál es el tratamiento dado a los resultados? 


\subsection{Recogida de datos}

La recogida de datos para la elaboración de este estudio se realizó a través del análisis de los informes de cuentas de las cooperativas seleccionadas. Conforme Yin (2009), se trata de una metodología de observación directa y de participación activa adecuada a este tipo de estudio.

\subsection{La muestra}

Actualmente, según los datos del INE portugués -“Conta Satélite de Economía Social 2010" (Instituto Nacional de Estatística), existen 2260 cooperativas en Portugal, en tipos de actividad económica distintos ya previstos en el Ccoop, cuyos porcentajes se presentan a continuación en el gráfico 1.

Gráfico 1. Ramas Cooperativas en Portugal en términos porcentuales

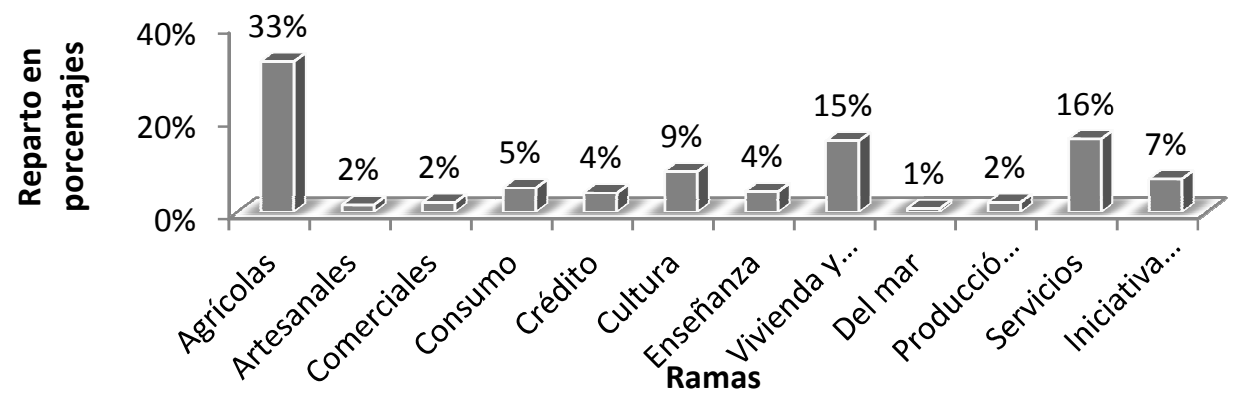

Fuente: Elaboración propia con base en el INE- "Conta Satélite de Economia Social de 2010"

De acuerdo con el gráfico 1, se observa que en Portugal las cooperativas agrícolas son las predominantes con el 33\%, seguida por las cooperativas de vivienda y construcción con el $15 \%$, después las cooperativas de servicios con el $16 \%$ y, en cuarto lugar surgen las de solidaridad e iniciativa social con el $7 \%$.

Con base en la información incluida en el gráfico 1, fueron escogidas para la muestra de este estudio cooperativas de las ramas más representativas, a saber:

- de la rama agrícola, Cave Santa Marta, CRL, Adega cooperativa de Favaios, CRL, y Adega cooperativa de Vila Real- Caves Vale do Corgo, CRL;

- de la rama de vivienda y construcción, Habece - cooperativa de habitação e construção de Cedofeita, CRL, y la cooperativa de vivienda económica Nova Morada CRL;

- de la rama de servicios, la cooperativa Mútua dos Pescadores; 
- de la rama de iniciativa social, la cooperativa de Educação e Reabilitação de Crianças Inadaptadas de Fafe, CRL (CERCIFAF) y la cooperativa de Educação e Reabilitação do Cidadão Inadaptado de Espinho, CRL (CerciEspinho).

Recogimos los informes de cuentas de las cooperativas seleccionadas, teniendo como período de análisis los años de 2010 a 2013.

\section{ANÁLISIS Y DISCUSIÓN DE LOS RESULTADOS}

A pesar del número elevado de cooperativas existentes en Portugal, tuvimos grandes dificultades en obtener los informes de cuentas, puesto que entramos en contacto con diversas cooperativas de diferentes ramas y no logramos una respuesta de muchas de estas. Por tanto, relativamente a los informes de cuentas de las cooperativas de vivienda y construcción, servicios y de iniciativa social, estos eran accesibles en las respectivas páginas de Internet. Respecto a los informes de las cooperativas agrícolas y de CerciEspinho, estos fueron gentilmente cedidos.

Los estados financieros de las cooperativas agrícolas, de las cooperativas de vivienda y construcción y de la cooperativa de iniciativa social CERCIFAF, fueron elaborados con base en la normativa contable vigente, el SNC, durante el período de 2010 a 2013.

La cooperativa de servicios Mutua dos Pescadores elaboró sus balances de cuentas según el Plan de Cuentas para Empresas de Seguros ${ }^{14}$.

Debemos señalar que de CerciEspinho estudiaremos solo las cuentas anuales del año 2012 (elaborado según el SNC) y del año 2013 (elaborado según el SNC-ESNL), puesto que los estados financieros hasta el año 2011 se elaboraron según el revocado Plan Oficial de Contabilidad para las Instituciones de Solidaridad Social ${ }^{15}$.

Los datos referentes a los resultados obtenidos por las cooperativas de la muestra fueron recogidos y analizados con el objetivo de comprobar cuál es el tratamiento dado a los respectivos resultados de los ejercicios en análisis.

A ese respecto, buscamos averiguar si las cuentas de resultados evidencian los diferentes tipos de resultados.

\footnotetext{
${ }^{14}$ Aprobado por la Norma Reglamentaria n. ${ }^{\circ}$ 4/2007-R, de 27 de Abril.

${ }^{15}$ Decreto Ley n. ${ }^{\circ} 78 / 89$ de 3 de Marzo.
} 
Cuadro 1. Cooperativas que revelan los diferentes tipos de resultados

\begin{tabular}{|l|l|c|c|}
\hline \multicolumn{1}{|c|}{ Ramas } & \multicolumn{1}{|c|}{ Cooperativas } & Sí & No \\
\hline \multirow{5}{*}{ Agrícola } & Caves Santa Marta & & $\mathbf{X}$ \\
\cline { 2 - 4 } & Adega cooperativa de Favaios & & $\mathbf{X}$ \\
\cline { 2 - 4 } & Adega Vila Real & & $\mathbf{X}$ \\
\hline \multirow{2}{*}{ Vivienda y construcción } & Habece & & $\mathbf{X}$ \\
\cline { 2 - 4 } & Nova Morada & & $\mathbf{X}$ \\
\hline Servicios & Mútua dos Pescadores & & $\mathbf{X}$ \\
\hline \multirow{2}{*}{ Iniciativa Social } & CERCIFAF & & $\mathbf{X}$ \\
\cline { 2 - 4 } & CerciEspinho & & $\mathbf{X}$ \\
\hline
\end{tabular}

Fuente: Elaboración propia

Según el cuadro 1, las cooperativas estudiadas no muestran los diferentes tipos de resultados cooperativos, ni refieren en los informes de gestión esa distinción, con excepción de Mútua de Pescadores que la presenta de forma bastante apreciable en su informe de gestión. Sin embargo, Mutua dos Pescadores, por ser una cooperativa relacionada con el área de los seguros, no adopta el SNC y sus estados financieros se prepararon con base en el plan especial de cuentas para las empresas de seguros.

Teniendo en cuenta que la mayoría de las cooperativas de nuestra muestra no distingue los diferentes tipos de resultados, a continuación pasaremos al análisis de las cooperativas estudiadas.

Cuadro 2. Aplicación de los resultados en las cooperativas en Portugal

\begin{tabular}{|c|c|c|c|}
\hline Ramas & Cooperativas & Resultado neto & Aplicación de los Resultados \\
\hline \multirow{4}{*}{ Agrícola } & \multirow{2}{*}{ Caves Santa Marta } & Positivo & Reservas \\
\hline & & Negativo & Resultados diferidos \\
\hline & Adega de Favaios & Positivo & Reservas \\
\hline & Adega Vila Real & Positivo & Reserva de Inversiones \\
\hline \multirow{2}{*}{$\begin{array}{l}\text { Vivienda y } \\
\text { Construcción }\end{array}$} & Habece & Positivo & Reservas \\
\hline & Nova Morada & Negativo & Resultados diferidos \\
\hline \multirow{3}{*}{ Servicios } & \multirow{3}{*}{ Mútua dos Pescadores } & Negativo & Resultados diferidos \\
\hline & & \multirow{2}{*}{ Positivo } & Transacciones con Cooperativistas \\
\hline & & & Transacciones con terceros \\
\hline \multirow{3}{*}{ Iniciativa Social } & \multirow{2}{*}{ CERCIFAF } & Negativo & Resultados diferidos \\
\hline & & Positivo & Resultados diferidos \\
\hline & CerciEspinho & Positivo & Reservas \\
\hline
\end{tabular}

Fuente: Elaboración propia 
Al analizar el cuadro 2, se constata que en la cooperativa Caves Santa Marta el resultado neto positivo del año 2010 es transferido a las reservas, puesto que la constitución de estas es obligatoria, según el Ccoop (arts. $96^{\circ}$ y 97). Por eso, en los informes de gestión no se menciona si los resultados fueron obtenidos como resultado de transacciones con cooperativistas o con terceros. A su vez, los resultados negativos en los años 2011, 2012 y 2013 son transferidos para la cuenta de resultados diferidos.

Adega de Favaios transfiere el resultado neto del período para la cuenta de reservas. Estas reservas son obligatorias y se encuentran previstas en los artículos $96^{\circ}$ y $97^{\circ}$ del Ccoop. Sin embargo, la cooperativa en causa no se refiere en ninguna ocasión al origen de esos resultados.

La bodega Adega Vila Real transfirió los resultados positivos a las reservas de inversiones, previstas en el régimen jurídico de las cooperativas agrícolas (art. $12^{\circ}$ del $\mathrm{DL} \mathrm{n}{ }^{\circ}$ 335/99 de 20 de agosto). Los estados financieros de la bodega cooperativa de Vila Real revelan que ya está debidamente constituida la reserva legal obligatoria.

Si analizamos los estados financieros de las cooperativas agrícolas, comprobaremos que estas no contabilizan separadamente las transacciones con los cooperativistas de las llevadas a cabo con terceros. Sin embargo, siguen el Ccoop y el reglamento de las cooperativas agrícolas en lo referido a la constitución de las reservas.

La cooperativa de vivienda y construcción Habece cumple el Ccoop y el régimen jurídico de las cooperativas de vivienda y construcción, ya que destina un porcentaje de los excedentes a la reserva de educación y formación cooperativa (art. 97 ${ }^{\circ}$ del Ccoop), a la reserva de conservación y reparación y a la reserva de construcción y cooperación, previstas en el art. $12^{\circ}$ del DL n ${ }^{\circ} 502 / 99$ de 19 de noviembre. El art. $15^{\circ}$ de dicho reglamento establece que los excedentes resultantes de transacciones con los miembros deberán ser destinados a reservas, por lo que en esta rama cooperativa no se permite el retorno de los excedentes.

La cooperativa Nova Morada ha presentado en los últimos 4 años un resultado neto negativo, transfiriéndolo a los resultados diferidos. Los estados financieros de Nova Morada no nos indican si esos resultados netos negativos se obtuvieron en transacciones con cooperativistas o con terceros. 
El análisis de los estados financieros de la cooperativa de servicios Mútua de Pescadores permitió comprobar que las transacciones con los cooperativistas y las realizadas con terceros se muestran de forma separada, conforme el detalle en el Anexo a los estados financieros, siguiendo de esta forma con rigor el Ccoop.

Con respecto a las cooperativas de iniciativa social, CERCICAF transfiere los resultados netos negativos de los años 2010 y 2011 a los resultados diferidos. A su vez, los resultados netos positivos correspondientes a los años 2012 y 2013 son también transferidos a los resultados para cubrir perjuicios de años anteriores.

CerciEspinho presenta resultado neto positivo. Ahora bien, el art. $7^{\circ}$ del DL n ${ }^{\circ}$ 7/98 de 15 de enero, relativo al régimen jurídico de las cooperativas de solidaridad e iniciativa social, exige que los excedentes existentes reviertan obligatoriamente en las reservas. Las reservas constituidas por CerciEspinho integran la reserva legal (art. $96^{\circ}$ del Ccoop), la reserva de educación y formación cooperativa (art $97^{\circ}$ del Ccoop), la reserva de inversión y la reserva de integración profesional de los alumnos (reservas previstas en los estatutos de la cooperativa).

Debemos destacar que al analizar el informe de gestión de CerciEspinho del año 2013, comprobamos que la terminología utilizada en virtud del cambio de normativa está más adecuada a la realidad de las cooperativas.

Conviene añadir que la cuenta 26 - Fundadores/ Beneméritos/ Donantes/ Asociados/ Miembros, del reglamento de cuentas propuesto por el SNC-ESNL, se diferencia claramente de la cuenta 26 -Accionistas/ Socios del código de reglamento de cuentas propuesto por el SNC.

De hecho, como vimos, las cooperativas no tienen socios sino cooperativistas, es decir, miembros que participan activamente en la actividad de la cooperativa.

Al analizar los estados financieros, se constata que el SNC-ESNL permitió a CerciEspinho transmitir una imagen realista y apropiada de su desempeño y, de esa forma, mostrar su verdadero objeto y finalidad: la mutualista.

Cabe subrayar que según el recién aprobado DL no 98/2015 de 2 de junio, las cooperativas de iniciativa social se equiparan a las entidades u organizaciones sin ánimo de lucro, por lo que deben adoptar el SNC-ESNL. 
En el transcurso del análisis realizado, se comprobó que el tratamiento contable de los resultados seguido por las cooperativas, que presentan sus estados financieros siguiendo el SNC, muestra algunas particularidades, con especial atención para: (i) la necesaria dotación de las reservas obligatorias (reserva legal; reserva de educación y formación cooperativa y otras reservas), (ii) la imposibilidad de repartir los resultados provenientes de transacciones con terceros, (iii) la existencia de diferentes tipos de resultados, teniendo en cuenta su origen (transacciones con cooperativistas o con terceros o en operaciones ajenas al objeto social de la cooperativa).

Así pues, para comprender mejor las especificidades del tratamiento contable de los resultados en las cooperativas, los hemos contrastado con el tratamiento de los resultados en las sociedades mercantiles, según consta en el cuadro 3.

El cuadro ha sido elaborado con base tanto en la observación práctica, a través del análisis de los informes de cuentas, como en la legislación existente sobre el tema, especialmente el Ccoop y el CSC, con la finalidad de subrayar las particularidades de las cooperativas frente a las sociedades mercantiles.

Cuadro 3.Análisis comparativo de los resultados entre cooperativas y sociedades comerciales

\begin{tabular}{|c|c|c|}
\hline Cooperativas & & Sociedades Mercantiles \\
\hline \multicolumn{3}{|c|}{ Elemento teleológico } \\
\hline \multicolumn{2}{|c|}{ Finalidad mutualista } & Fines lucrativos \\
\hline \multicolumn{3}{|c|}{ Reservas } \\
\hline \multicolumn{2}{|c|}{$\begin{array}{c}\text { Arts. } 96^{\circ}, 97^{\circ} \text { e } 98^{\circ} \text { del CCoop } \\
\text { Reserva Legal + Reserva Educación y formación } \\
+ \text { Otras Reservas }\end{array}$} & $\begin{array}{l}\text { Arts. } 218^{\circ}, 295^{\circ}, 296^{\circ} \mathrm{CSC} \\
\text { Reserva Legal+ Reserva Estatutaria }+ \\
\text { Reserva libre }\end{array}$ \\
\hline \multicolumn{3}{|c|}{ Resultados } \\
\hline $\begin{array}{c}\text { Transacciones con } \\
\text { cooperativistas }\end{array}$ & $\begin{array}{l}\text { Retorno } \\
\text { Art. } 100^{\circ} \mathrm{n} .^{\circ} 1 \text { CCoop }\end{array}$ & \multirow{2}{*}{$\begin{array}{l}\text { Beneficios /Dividendos } \\
\text { Arts. } 217^{\circ} \text { e } 294^{\circ} \mathrm{CSC}\end{array}$} \\
\hline $\begin{array}{c}\text { Transacciones con } \\
\text { Terceros }\end{array}$ & $\begin{array}{l}\text { Beneficios/ Resultados } \\
\text { Irrepartibles - Art. } 99^{\circ}\end{array}$ & \\
\hline \multicolumn{3}{|c|}{ Distribución de los Resultados } \\
\hline \multicolumn{2}{|c|}{$\begin{array}{c}\text { Excedentes -proporcionales a las transacciones realizadas } \\
\text { por cada miembro } \\
\text { Beneficios - no pueden ser distribuidos (afectado a la } \\
\text { reserva obligatoria e irrepartibles) }\end{array}$} & $\begin{array}{c}\text { Dividendos -proporcionales a la parte del capital social } \\
\text { perteneciente a cada socio }\end{array}$ \\
\hline \multicolumn{3}{|c|}{ Pérdidas/ Deudas } \\
\hline \multicolumn{2}{|c|}{$\begin{array}{c}\text { Pérdidas - Se podrán imputar a los cooperativistas } \\
\text { Resultados diferidos }\end{array}$} & Resultados diferidos \\
\hline
\end{tabular}

Fuente: Elaboración propia 
De acuerdo con el cuadro 3, las cooperativas presentan diferencias sustanciales con relación a las sociedades mercantiles en lo que respecta al tipo de resultados y a su distribución.

Tal y como hemos ya mencionado, las cooperativas pueden realizar transacciones con cooperativistas y con terceros. En las transacciones con los cooperativistas, los resultados cooperativos positivos -excedentes- pueden retornar al cooperativista según el $\operatorname{art} .100^{\circ} \mathrm{n}^{\mathrm{o}} 1$ del Ccoop, ya que la distribución de esos excedentes se hace según la proporción de las transacciones realizadas por cada uno de los miembros con la cooperativa. En las transacciones con terceros, los resultados son considerados beneficios, los cuales son transferidos a las reservas obligatorias e irrepartibles. A su vez, en las sociedades mercantiles todos los resultados positivos son considerados beneficios, que cuando se reparten los denominamos dividendos y que, por normal general, se distribuyen de forma proporcional a la parte del capital perteneciente a cada socio (art. $22^{\circ}$ del CSC).

Los datos presentados confirman que las cooperativas presentan un marco contable poco adecuado, puesto que los estados financieros no están ajustados a la realidad de las mismas. Ante esta situación responderemos a las cuestiones inicialmente propuestas.

P1: ¿La cuenta de resultados pone de manifiesto los diferentes tipos de resultados?

A lo largo de este estudio se constató que los estados financieros de las cooperativas seleccionadas no muestran adecuadamente los diferentes tipos de resultados según la naturaleza y especificidad de las cooperativas.

De las cooperativas analizadas, solo la cooperativa Mútua dos Pescadores organiza su contabilidad de forma diferenciada, de ahí que lo hagan con base en un porcentaje, o sea, los resultados provenientes de terceros no son resultado de las transacciones efectivamente realizadas sino de un porcentaje de ellas.

P2: ¿Cuál es el tratamiento dado a los resultados?

Con respecto a los excedentes, las cooperativas los transfirieron a las reservas previstas en las diferentes normativas jurídicas. Constatamos que la cooperativa agrícola Adega de Favaios transfiere sus excedentes a la reserva legal, a la reserva de educación y formación cooperativa y a la reserva libre, mientras que la cooperativa Habece de la rama de vivienda y construcción los transfiere a la reserva de educación y formación cooperativa, a la 
de conservación y reparación, a la de construcción y, por último, también a la reserva cooperativa; la cooperativa de iniciativa social CerciEspinho transfiere sus excedentes a la reserva legal, a la de educación y formación cooperativa, a la de inversiones y, por último, a la reserva de integración profesional de los alumnos.

Las pérdidas fueron transferidas a los resultados diferidos.

\section{CONSIDERACIONES FINALES}

Las cooperativas tienen como objeto y finalidad principal la mutualista, o sea, la actividad está dirigida a la satisfacción de las necesidades económicas y sociales de los cooperativistas, ya que estos participan en dicha actividad (actividad cooperativizada).

Ello explica que los resultados cooperativos positivos de las cooperativas sean en su mayoría excedentes, los cuales, cuando se reparten son considerados retorno. Los resultados negativos provenientes de las transacciones con cooperativistas se denominan pérdidas, pudiendo estas ser imputadas al cooperativista de forma proporcional a su participación en la actividad de la cooperativa.

Los resultados provenientes de las transacciones con terceros (resultados extracooperativos) si son positivos consisten en beneficios y si son negativos se traducen en perjuicios.

Los resultados provenientes de actividad ajena al objeto social de la cooperativa (resultados extraordinarios), si son positivos son beneficios y si son negativos se denominan perjuicios.

Estos beneficios no pueden ser objeto de reparto entre los cooperativistas, viéndose obligatoriamente afectadas las reservas irrepartibles.

En este estudio de caso se identificaron transacciones con cooperativistas, así como con terceros, pero los estados financieros no permiten comprobar los resultados positivos provenientes de los dos tipos de operaciones, respectivamente excedentes y beneficios. Por otro lado, los estados financieros tampoco permiten mostrar los tipos de resultados negativos que son identificables en las cooperativas (deudas y pérdidas). 
Asimismo, las cooperativas no tienen ánimo de lucro y, por esa causa, la cuenta de resultados debería mostrar una mayor transparencia en la cálculo de los resultados, así como la información divulgada debería velar por los intereses específicos de los principales clientes y entidades con las que mantienen algún tipo de relación.

Se constató que al abrigo del SNC las cooperativas son tratadas como si fuesen sociedades mercantiles, lo que no permite mostrar las especificidades de sus resultados positivos y negativos. Por eso la normativa aplicable a las ESNL es una normativa más específica y adecuada a la tipología de los resultados presentes en las cooperativas, ya que esta permite que en la cuenta de resultados se discrimine el resultado neto del ejercicio según las diversas actividades.

Después de identificar los diferentes tipos de resultados de las cooperativas de la muestra seleccionada, que en futuras investigaciones intentaremos ampliar, de comprender el tratamiento jurídico y contable de los resultados, concluimos que la actual normativa contable en vigor no permite constatar las especificidades de los resultados de las cooperativas en general. Así pues, consideramos que estas entidades deberían regirse según el SNC-ESNL, por tratarse de una normativa más específica y adecuada a la realidad de las cooperativas para que sus estados financieros puedan proporcionar una imagen fiel y apropiada de su desempeño.

\section{BIBLIOGRAFÍA}

AGUIAR, N. (2014) A tributação dos rendimentos das cooperativas em Portugal Cooperativismo e Economia Social, $\mathrm{n}^{\circ}$ 36, pp. 55-80.

BANDEIRA, A. y MEIRA, D. (2015) A inadequação do enquadramento contabilístico das cooperativas em Portugal (I). Revista OTOC, nº 179, pp.42-47.

CARRERAS ROIG, L. (2011) Consideraciones en torno al posible carácter mercantil de las sociedades cooperativas y acerca de las limitaciones a la realización de operaciones con terceros no socios de estas entidades. REVESCO. Revista de Estudios Cooperativos, Tercer Cuatrimestre, $\mathrm{n}^{\circ}$ 106, pp. 55-73. DOI:

http://dx.doi.org/10.5209/rev_REVE.2011.v106.37378.

J. DOMINGUES, P. (2012) Código das Sociedades Comerciais em Comentário. (coord. de Jorge Manuel Coutinho de Abreu). Coimbra: Almedina. 
DONÁRIO, A. (2010) Natureza dos excedentes e reservas nas cooperativas: seu retorno e distribuição. Centro de Análise Económica de Regulação Social. Universidade Autónoma de Lisboa. Versão Eletrónica. Acedido em 8 de Dezembro de 2014, disponível em: http://www.universidade-autonoma.pt/upload/galleries/excedentes-para-cars.pdf.

FAJARDO GARCÍA, I. (1997) La gestión económica de la cooperativa: Responsabilidad de los socios. Madrid: Editorial Tecnos.

FRAGA, M. y GONÇALVES, D. A. (2009) Acão ut singuli (de responsabilidade civil) e a relação do Direito Cooperativo com o Direito das Sociedades Comerciais, Revista de Direito das Sociedades, Ano I (2009), n 4, Almedina, pp. 888-904.

INE (2013) Conta satélite da economia social 2010. Lisboa-Portugal: Instituto Nacional de Estatística, I.P. e CASES, Cooperativa António Sérgio para a Economia social, CIPRL. Versão Eletrónica. Acedido em 21 de dezembro de 2014, disponível em: https://www.ine.pt/xportal/xmain?xpid=INE\&xpgid=ine_publicacoes\&PUBLICACOESp $\underline{\mathrm{ub} \text { boui }=157543613 \& \text { PUBLICACOESmodo=2 }}$.

ITURRIOZ, J (1999) El resultado de las sociedades cooperativas y su distribución en la nueva Ley de Cooperativas 27/1999. REVESCO. Revista de Estudios Cooperativos, n. ${ }^{\circ}$ 69, pp. 128-149.

LOPES, L. (2015) Breves nótulas sobre o "Novo Estatuto" das Instituições Particulares de Solidariedade Social do Direito Nacional e no Direito da União Europeia. Cooperativismo

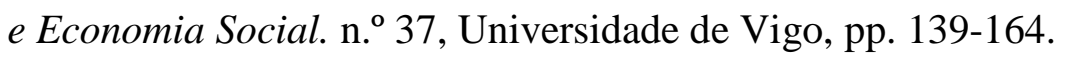

MARÍN-SÁNCHEZ, et al. (2012) Las pérdidas en las cooperativas: Análisis de la legislación substantiva, contable y fiscal. Barcelona: Marcial Pons.

MEIRA, D. (2010) As operações com terceiros no direito cooperativo português. Comentário ao Acordão do Supremo Tribunal de Justiça de 18 de dezembro de 2007. Ciências Empresariais e Jurídicas, n. ${ }^{\circ}$ 17, ISCAP, pp. 93-112.

MEIRA, D. (2011) As insuficiências do regime legal do capital social e das reservas na cooperativa. I Congresso do Direito das Sociedades em Revista. Almedina, Coimbra, pp. 129-155.

MEIRA, D. (2012a) O regime jurídico do excedente Cooperativo. In: Jurisprudência Cooperativa Comentada. Obra Coletiva de comentários e acórdãos da jurisprudência portuguesa, brasileira e espanhola. Lisboa: Imprensa Nacional-Casa da Moeda, pp. 359374.

MEIRA, D. (2012b) As Operações com terceiros no direito cooperativo português. In: Jurisprudência Cooperativa Comentada. Obra Coletiva de comentários e acórdãos da 
jurisprudência portuguesa, brasileira e espanhola. Lisboa: Imprensa Nacional- Casa da Moeda, pp. 413-425.

MEIRA, D. (2012c) Revisitando o problema da distinção entre excedente cooperativo e lucro societário. In: II Congresso do Direito das Sociedades em Revista, pp. 353-374.

MEIRA, D. (2015) Da possibilidade de imputação de perdas ao cooperador numa régie cooperativa. Cooperativismo e Economia Social, n. ${ }^{\circ}$ 37, Universidade de Vigo, pp. 63-89.

MEIRA, D. y RAMOS, M. (2014) Governação e regime económico das cooperativas-Estado da arte e linhas de reforma. Porto: Vida Económica.

NAMORADO, R. (2005) Cooperatividade e Direito Cooperativo, estudos e pareceres. Coimbra: Almedina.

RODRIGUES, A. (2010) Os novos desafios da contabilidade para organizações da economia social que apliquem o SNC às cooperativas. Revista Cooperativismo e Economia Social, n. ${ }^{\text {3 }}$ 2, Curso 2009/2010, Universidade de Vigo, pp. 115-140.

ROMERO CIVERA, A. y MARÍ VIDAL, S. (2015) Algunos aspectos críticos en la aplicación del impuesto de sociedades a las cooperativas según el régimen fiscal de cooperativas. La necesidad de su reforma. REVESCO. Revista de Estudios Cooperativos, Segundo Cuatrimestre, n. ${ }^{\circ} 118$, pp.7-30. DOI:

http://dx.doi.org/10.5209/rev_REVE.2015.v118.48709.

SGECOL (Study Group on European Cooperative Law), Draft Principles of European Cooperative Law (draft PECOL), May 2015, pp. 83-86. Acedido em 07 de junho de 2015, disponível em http://www.euricse.eu/wp-content/uploads/2015/04/PECOL-May-2015.pdf.

VARGAS VASSEROT, C. (2006) La actividad cooperarativizada y las relaciones de la cooperativa com sus sócios y com terceros Navarra: Editorial Aranzadi.

YIN, R. (2009) Case Study Research: Design and Methods. $4^{\mathrm{a}}$ ed., London: Sage Publications.

\section{Legislación}

Aviso n. ${ }^{\circ} 15652 / 2009$ de 7 de setembro. Diário da República n. ${ }^{\circ}$ 173/2009 - II Série. Secretário-Geral. Lisboa.

Decreto-Lei n.. 7/98, de 15 de janeiro. Diário da República $n .^{\circ}$ 12/1998 - I Série A. Ministério do Trabalho e da Solidariedade. Lisboa.

Decreto-lei n. ${ }^{\circ}$ 158/2009 de 13 de julho. Diário da República n. ${ }^{\circ}$ 133/2009 - I Série. Ministério das Finanças e da Administração Pública. Lisboa.

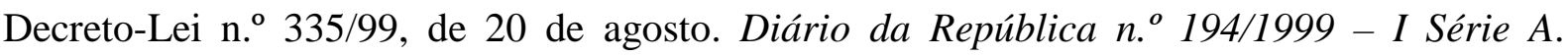
Ministério da Agricultura desenvolvimento Rural e Pescas. Lisboa. 
Decreto-Lei n. ${ }^{\circ}$ 502/99, de 19 de novembro. Diário da República n. ${ }^{\circ}$ 270/1999 - I Série A. Ministério do Trabalho e da Solidariedade. Lisboa.

Decreto-Lei n. ${ }^{\circ}$ 36-A/2011 de 9 de março. Diário da República n. ${ }^{\circ}$ 48/2011 - I Série. Ministério das Finanças e da Administração Pública. Lisboa.

Decreto-Lei n..$^{\circ}$ 98/2015 de 2 de junho. Diário da República n. ${ }^{\circ}$ 106/2015 - I Série. Ministério das Finanças e da Administração Pública. Lisboa.

Diretiva n. ${ }^{\circ}$ 91/674/CEE de 19 de dezembro. Jornal Oficial das Comunidades Europeias L374/7 de 19 de dezembro de 1991. Parlamento Europeu e do Conselho. Bruxelas.

Lei n. ${ }^{\circ}$ 85/98 de 16 de dezembro. Diário da República n. ${ }^{\circ}$ 289/1998 - I Série. A Assembleia da República. Lisboa.

Lei Constitucional n. ${ }^{\circ}$ 1/2005 de 12 de agosto. Sétima revisão constitucional. Diário da República n. ${ }^{o}$ 155/2005 - I Série. Assembleia da República. Lisboa.

Lei n. ${ }^{\circ}$ 64-B/2011 de 30 de dezembro. Diário da República n. ${ }^{\circ}$ 250/2011 - I Série. Suplemento. Assembleia da República. Lisboa.

Lei n. ${ }^{\circ}$ 119/2015 de 31 de agosto. Diário da República n. ${ }^{\circ}$ 169/2015 - I Série. Assembleia da República. Lisboa.

Ley n. ${ }^{\circ}$ 27/1999, de 16 de julio - Boletín Oficial del Estado n. ${ }^{\circ}$ 27/1999. I Sección. Jefatura del Estado. Madrid.

Portaria n. ${ }^{\circ}$ 218/2015 de 23 de julho. Diário da República n. ${ }^{\circ}$ 142/2015 - I Série. Ministério das Finanças. Lisboa. 